\title{
The I-mode confinement regime at ASDEX Upgrade: global properties and characterization of strongly intermittent density fluctuations
}

\author{
T. Happel, ${ }^{1,}{ }^{*}$ P. Manz,${ }^{1,2}$ F. Ryter, ${ }^{1}$ M. Bernert, ${ }^{1}$ M. Dunne, ${ }^{1}$ P. Hennequin, ${ }^{3}$ A. Hetzenecker, ${ }^{1}$ \\ U. Stroth, ${ }^{1,2}$ G. D. Conway, ${ }^{1}$ L. Guimarais,${ }^{4}$ C. Honore, ${ }^{3}$ E. Viezzer ${ }^{\dagger},{ }^{1}$ and the ASDEX Upgrade Team ${ }^{1}$ \\ ${ }^{1}$ Max-Planck-Institut für Plasmaphysik, Boltzmannstr. 2, 85748 Garching, Germany \\ ${ }^{2}$ Physik-Department E28, Technische Universität München, James-Franck-Str. 1, 85748 Garching, Germany \\ ${ }^{3}$ Laboratoire de Physique des Plasmas, Ecole Polytechnique, 91128 Palaiseau, France \\ ${ }^{4}$ Instituto de Plasmas e Fusão Nuclear, Instituto Superior Técnico, Universidade de Lisboa, Lisbon, Portugal
}

(Dated: 2017-01-26)

\begin{abstract}
Properties of the I-mode confinement regime on the ASDEX Upgrade tokamak are summarized. A weak dependence of the power threshold for the L-I transition on the toroidal magnetic field strength is found. During improved confinement, the edge radial electric field well deepens. Stability calculations show that the I-mode pedestal is peeling-ballooning stable. Turbulence investigations reveal strongly intermittent density fluctuations linked to the weakly coherent mode in the confined plasma, which become stronger as the confinement quality increases. Across all investigated structure sizes $\left(k_{\perp} \approx 5-12 \mathrm{~cm}^{-1}\right.$, with $k_{\perp}$ the perpendicular wavenumber of turbulent density fluctuations), the intermittent turbulence bursts are observed. Comparison with bolometry data shows that they move poloidally toward the X-point and finally end up in the divertor. This might be indicative that they play a role in inhibiting the density profile growth, such that no pedestal is formed in the edge density profile.
\end{abstract}

\section{INTRODUCTION}

Improved confinement regimes are a crucial part of magnetic confinement fusion research due to their effectiveness in obtaining high plasma stored energies at comparably low heating levels compared to low confinement regimes [1-3]. While the high confinement mode (H-Mode) is characterized by strong plasma edge gradients in both density and temperature, the so-called pedestal, the improved energy confinement mode (I-mode) $[3,4]$ is made distinctive by the fact that a pedestal only builds up in the temperature and not the density.

Owing to this selective confinement improvement, the Imode has some unique properties: first, the impurity confinement time is low due to the absence of a particle transport barrier [3], resulting in low values of the effective charge $Z_{\text {eff }}$. Second, the I-mode does not develop edge-localize modes (ELMs) [5, 6], which makes it attractive from the point of view of divertor power loads [7]. Other features of the Imode are that it can only be accessed if the power threshold to obtain the H-mode $P_{\mathrm{L}-\mathrm{H}}$ is kept high, which can be achieved by using unfavorable magnetic configurations or hydrogen plasmas $[8,9]$. It has been observed on Alcator C-Mod that the power threshold to access the I-mode from L-mode $P_{\mathrm{L}-\mathrm{I}}$ depends less on the toroidal magnetic field strength $B_{t}$ than $P_{\mathrm{L}-\mathrm{H}}[10]$, which is nearly linearly dependent on $B_{t}$. Furthermore, the I-mode is characterized by an edge instability called the weakly coherent mode (WCM), which is coupled to the geodesic acoustic mode (GAM) $[11,12]$. The I-mode confinement regime has been reported from Alcator C-Mod, ASDEX Upgrade and DIII-D [3, 13, 14], while other devices have experiments scheduled [10].

${ }^{\dagger}$ present address: Dept. of Atomic, Molecular and Nuclear Physics, University of Seville, 41012 Seville, Spain

*Electronic address: tim.happel@ipp.mpg.de
Questions regarding the I-mode remain on the fact that an energy transport barrier is formed while particle transport stays L-mode like. Furthermore, the underlying instability of the WCM and its importance for confinement have not yet been resolved.

This paper reports on recent I-mode results from the ASDEX Upgrade tokamak (AUG). In section II, access conditions and the confinement improvement are documented along with peeling-ballooning stability considerations. Section III presents the edge density turbulence behavior, which is shown to be strongly intermittent and linked to the WCM, and also observed by other diagnostics. A summary is given in Sec. IV.

\section{ACCESS, CONFINEMENT AND STABILITY}

As stated above, the threshold power to access the I-mode $P_{\mathrm{L}-\mathrm{I}}$ has been observed to be linearly dependent on density [15] and only weakly dependent on the toroidal magnetic field strength $B_{t}$ [10]. The power considered here is $P_{\text {loss }}=P_{\text {heat }}-\mathrm{d} W / \mathrm{d} t$, with $P_{\text {heat }}$ the absorbed heating power and $W$ the plasma stored energy.

To investigate the parameter dependence of $P_{\mathrm{L}-\mathrm{I}}$ on AUG, slow power ramps have been carried out in discharges at different densities and magnetic field strengths $\left(\left|B_{t}\right|=1.8-\right.$ $3.0 \mathrm{~T})$. The transition to I-mode is detected by the appearance of the WCM in the power spectrum obtained from the conventional reflectometer measurement [16]. Configurations in upper single null (USN) and reversed $B_{t}$ lower single null (LSNrev) have been used, both having the ion $\nabla B$ drift pointing away from the active $\mathrm{x}$-point, hence are unfavorable configurations for $\mathrm{H}$-mode access, as required to access the Imode.

Figure 1(a) shows the dependence of $P_{\mathrm{L}-\mathrm{I}}$ on the lineaveraged core density for both configurations and various toroidal magnetic field strengths. Plasma currents are in the 

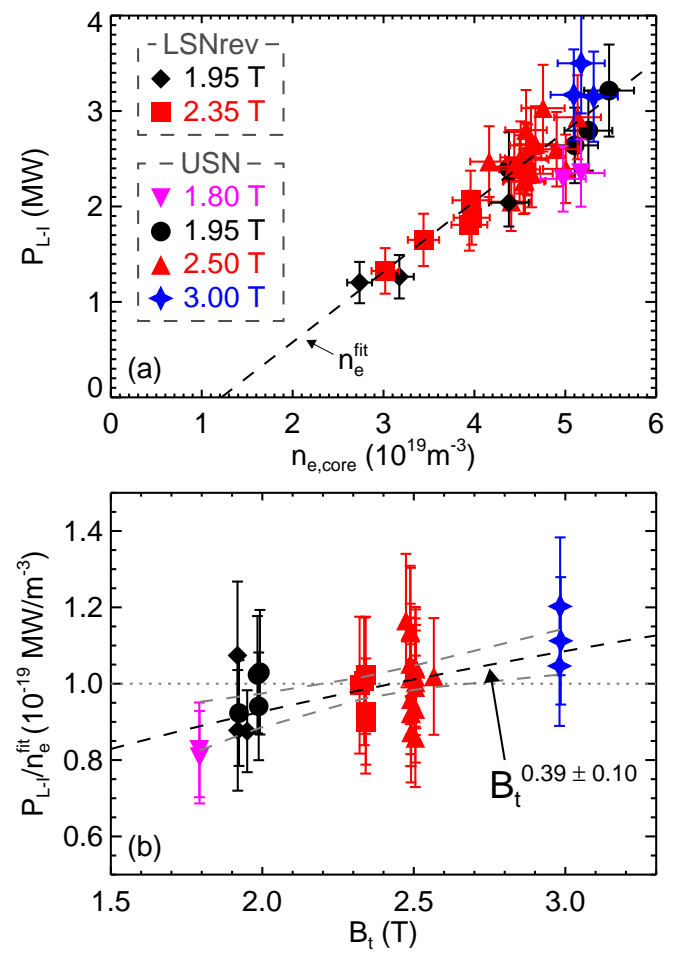

FIG. 1: (a) Power threshold $P_{\mathrm{L}-\mathrm{I}}$ for the transition from L-mode to Imode in AUG. An offset linear dependence on core density $\left(n_{e, \text { core }}\right)$ is observed $\left(n_{e}^{\mathrm{fit}}\right)$. (b) The removal of the density dependence $\left(P_{\mathrm{L}-\mathrm{I}} / n_{e}^{\mathrm{fit}}\right)$ reveals a weak dependence of $P_{\mathrm{L}-\mathrm{I}}$ on the toroidal magnetic field strength $B_{t}$.

range of $I_{p}=0.8-1.0 \mathrm{MA}$, which is a small variation $( \pm 11 \%)$ compared to the variation in $B_{t}( \pm 25 \%)$. Although there is a significant variation in magnetic field strength and equilibrium configuration (LSNrev / USN), the points show small scatter. An offset-linear dependence is observed. The linear fit $n_{e}^{\text {fit }}=0.737 n_{e \text {, core }}-0.896\left(n_{e}^{\mathrm{fit}}, n_{e, \text { core }}\right.$ in $10^{19} \mathrm{~m}^{-3}$, dashed line) has been obtained by fitting only the $2.35 \mathrm{~T} \mathrm{LSNrev}$ and 2.50 T USN points and will be used to account for the density dependence of $P_{\mathrm{L}-\mathrm{I}}$ in the following analysis.

The threshold power for the L-I transition normalized to the fit obtained above, $P_{\mathrm{L}-\mathrm{I}} / n_{e}^{\mathrm{fit}}$, is depicted in Fig. 1(b). In this representation, the points used to fit the data from Fig. 1(a) $(2.35-2.50 \mathrm{~T})$ scatter around unity. The dependence of $P_{\mathrm{L}-\mathrm{I}}$ on $B_{t}$ can thus be observed if a significant portion of points at other magnetic field strenghts lie above or below unity. This is the case for $B_{t}<2 \mathrm{~T}$, where most of the points are below unity. In contrast, all the points at $B_{t} \approx 3.0 \mathrm{~T}$ are above unity. A power law fit is shown as the dashed line, which has a dependence of $B_{t}^{0.39 \pm 0.10}$ ( $1 \sigma$ confidence interval), and is thus statistically significant. The $95 \%$ confidence interval is shown as grey dashed lines above and below the fit. Any trend that could fit into this interval would be $B_{t^{-}}$ dependent. The same conclusion is obtained if a regression is done with all data points of the form $P_{\mathrm{L}-\mathrm{I}} \propto n_{e}^{\alpha} B_{t}^{\beta}$, yielding $P_{\mathrm{L}-\mathrm{I}}=0.26 n_{e}^{1.36} B_{t}^{0.39}$. Inside the available density range, both offset-linear fit and regression give comparable values for $P_{\mathrm{L}-\mathrm{I}}$. However, an extrapolation to larger densities would yield a considerably different result. Experiments to extend the dataset toward higher densities than in Fig. 1(a) are scheduled to clarify this point. The results above indicate that there is indeed a weak magnetic field strength dependence of $P_{\mathrm{L}-\mathrm{I}}$ in AUG, in agreement with results from Alcator C-Mod [10]. It should be noted that this dependence is substantially weaker than the one for the $\mathrm{L}-\mathrm{H}$ transition, which is $B_{t}^{0.80 \pm 0.03}$ [17]. Furthermore, these results show that the density dependence of the L-I transition, which is at least linear, is slightly stronger than the density dependence of the high-density branch of the L-H transition $\left(P_{\mathrm{L}-\mathrm{H}} \propto n_{e}^{0.72}\right.$, see Ref. [17]).

The results presented above indicate that the magnetic field strength does indeed play a role in the L-I transition. However, based on this observation, it is unclear if and to what extent the L-I transition has commonalities with the well-characterized L-H transition, where $P_{\mathrm{L}-\mathrm{H}} \propto B_{t}^{0.8}$. Nevertheless, both I-mode and $\mathrm{H}$-mode owe their improved confinement to strong edge pedestals. Since at the L-H transition, pedestal dynamics and turbulence suppression play a major role, it is important to look in detail into the L-I transition and the fluctuations of the subsequent I-mode, which is done in the following.

Typical time traces of a discharge at $B_{t}=2.5 \mathrm{~T}$ going from L-mode through I-mode (starts at $2.23 \mathrm{~s}$ ) and into H-mode (at $2.59 \mathrm{~s}$ ) are presented in Fig. 2. In (a), the auxiliary heating is shown. During the whole time window, a central electron cyclotron resonance heating (ECRH, dashed) power of $750 \mathrm{~kW}$ is applied. At $2.1 \mathrm{~s}$, one neutral beam injection (NBI, solid black) source is added. The power $P_{\text {loss }}$ is shown in purple. After the NBI is switched on, $P_{\text {loss }}$ increases. Panel (b) depicts the core and edge line-average densities, which increase slightly when the NBI is added, but show little variation during I-mode. The edge ion and electron temperatures $T_{i}$ and $T_{e}$ and their gradients $-\nabla T_{i}$ and $-\nabla T_{e}$ are depicted in panels (c) and (d). After having almost reached saturation in the L-mode following the turn-on of the NBI, both $T_{i, e}$ and $-\nabla T_{i, e}$ increase throughout the whole time window of the I-mode, which results in the well-known edge pedestal in these quantities. The rate of increase changes abruptly at roughly $2.59 \mathrm{~s}$, when the plasma enters into H-mode. Figure 2(e) shows the confinement improvement factor $H_{98}(y, 2)$, which is usually below 0.6 for L-mode and above 0.8 in $\mathrm{H}$-mode. In the I-mode phase of the discharge, it increases up to values of $H_{98}(y, 2) \approx 0.85$. Since the normalized gradients $\left(R / L_{T e, T i}\right)$ of the core profiles remain unchanged due to profile stiffness, the edge pedestal is responsible for the higher core temperatures and thus for the significant increase in $H_{98}(y, 2)$. This increase is accompanied by a deepening of the edge radial electric $E_{r}$ field minimum, shown as solid points. More information on $E_{r}$ is presented later. The appearance of the WCM is depicted in Figs. 2(f-h). They show edge frequency spectra $\left(\rho_{\text {pol }}=0.997\right.$, with $\rho_{\text {pol }}$ the normalized poloidal flux radius) from the conventional reflectometer [16]. While the L-mode spectrum (f) exhibits a flat part at low frequencies up to about $50 \mathrm{kHz}$ and then a spectral fall-off, the I-mode spectra $(\mathrm{g})$ and $(\mathrm{h})$ show the WCM. At the beginning of the I-mode the WCM is barely visible above the broadband turbulence spectrum $(\mathrm{g})$, but it can achieve a considerable power above the background when the I-mode 


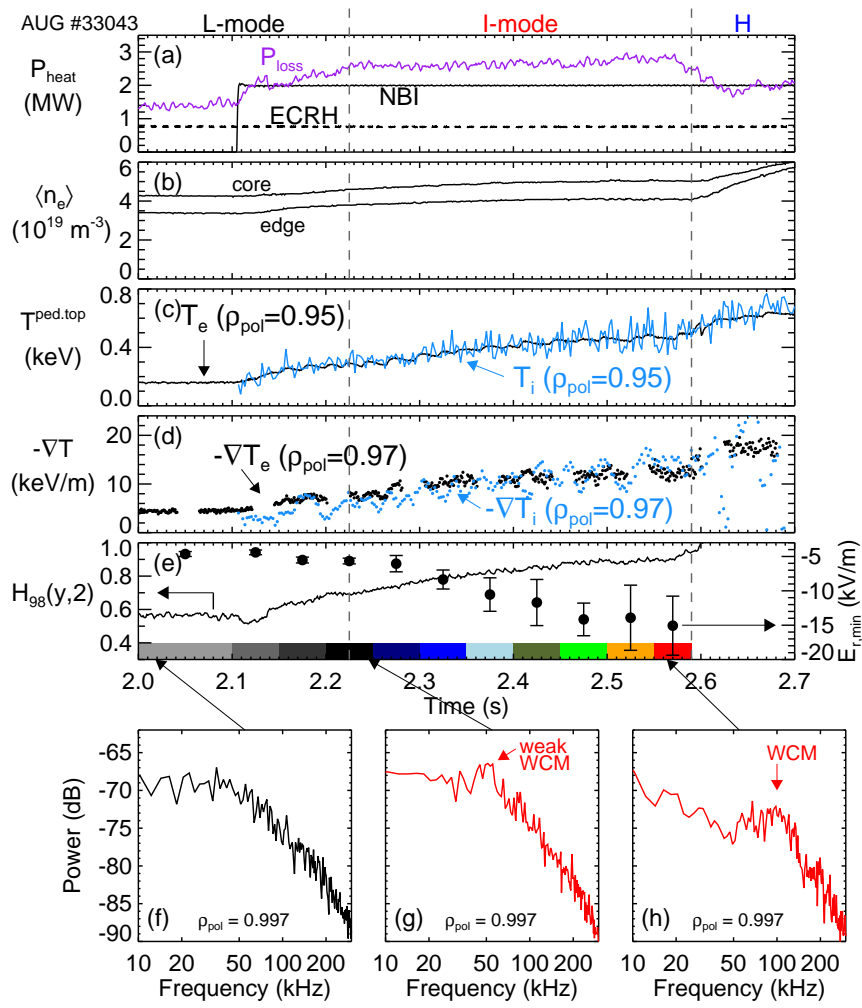

FIG. 2: Time traces of AUG \#33043, which goes from L-mode through I-mode into H-mode (indicated on top). (a) heating power, (b) line-averaged electron densities, (c) pedestal top electron and ion temperatures, (d) edge electron and ion temperature gradients, and (e) confinement factor $H_{98}(y, 2)$ and radial electric field minimum value. (f-h) Density fluctuation spectra measured at the times indicated by the arrows show the appearance of the WCM in I-mode. The colored blocks in (e) mark the time windows when $E_{r}$ profiles are measured (cf Fig. 3).

reaches a high $H_{98}(y, 2)$, which is usually close to the I-H transition (Fig. 2(h)). For this discharge the reflectometer was in frequency stepping mode to obtain radial fluctuation profiles in $50 \mathrm{~ms}$ time windows. Since the WCM is radially localized, the time point at which the WCM is first observed is not necessarily the time instant when it first actually appears. The plasma may have entered the I-mode up to $50 \mathrm{~ms}$ before $2.23 \mathrm{~s}$.

During the confinement improvement in I-mode, the aforementioned pedestal has a pronounced effect on the radial profile of the radial electric field $E_{r}$ due to the change in $\nabla p_{i}$, as can be observed in Fig. 2(e) and in more detail in Fig. 3. All profiles were inferred from the measurement of the perpendicular propagation velocity of density fluctuations $v_{\perp}$ with Doppler reflectometry via $E_{r}=\mathrm{v}_{\perp} B$. The assumption of a negligible phase velocity of the turbulence $\mathrm{v}_{\mathrm{ph}}$ compared to the $E \times B$ velocity, $\mathrm{v}_{\mathrm{ph}} \ll \mathrm{v}_{E \times B}$, has been used. This assumption has been shown to be valid in the edge region of the plasma [18-22], while some exceptions have been reported from the core [23-25]. Since the results presented here are from the edge plasma, the assumption is justified.

In Fig. 3, eleven radial $E_{r}$ profiles are shown. The color

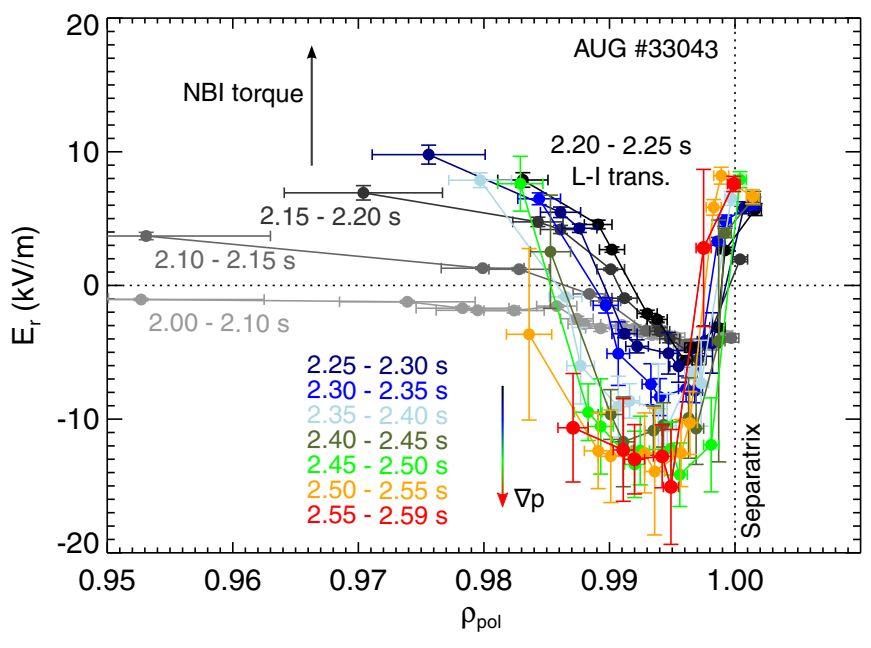

FIG. 3: Evolution of the edge radial electric field $\left(E_{r}\right)$ profile in Lmode $(2.00-2.25 \mathrm{~s})$ and throughout I-mode $(2.25-2.59 \mathrm{~s})$. At $t=2.59 \mathrm{~s}$, the transition to H-mode takes place. The effects of NBI torque input and the ion pressure gradient on $E_{r}$ are opposite and indicated by the arrows.

of each profile marks its respective time window, indicated by colored blocks in Fig. 2(d). The profile at $2.00-2.10 \mathrm{~s}$ is taken before the NBI is switched on (cf Fig. 2). At $2.10 \mathrm{~s}$, the NBI is switched on, which results in an increase in toroidal rotation, and according to radial force balance [26], in a successively more positive $E_{r}$ (indicated by arrow "NBI torque") in the core region until $2.25 \mathrm{~s}$. As the minimum of $E_{r}$ stays roughly constant at $-4.5 \mathrm{kV} / \mathrm{m}$, this results in an increased $E_{r}$ shear at $\rho_{\text {pol }} \approx 0.99$. The first $E_{r}$ profile to show a significantly deeper minimum is observed right after the I-mode has set in, at $2.30-2.35 \mathrm{~s}$. If the radial electric field shear plays a role in the L-I transition, the above suggests that it is the inner (negative) shear of the $E_{r}$ well which governs the dynamics, since the outer (positive) shear does not change. Subsequently, the edge $E_{r}$ profile becomes deeper as the I-mode, i.e. $\nabla p_{i}$ (indicated by arrow in Fig. 3), evolves. At $2.59 \mathrm{~s}$, the $E_{r}$ minimum is about $-14 \mathrm{kV} / \mathrm{m}$, and the H-mode starts. It should be noted that although the $E_{r}$ shear might be different, the value of $E_{r, \text { min }} \approx-14 \mathrm{kV} / \mathrm{m}$ is comparable to the value obtained for L-H transitions in favorable configurations [27]. The slow evolution of the edge $E_{r}$ well is correlated with the slow evolution of the pedestal and in consequence the confinement improvement factor $H_{98}(y, 2)$. This is in agreement with results that show that a sheared radial electric field has substantial impact on turbulence (see Ref. [28-30] and references therein) and thus can contribute to obtain stronger pedestals. Assuming a constant width of the $E_{r}$ well, its depth is a proxy for the flow shear [22], which will be investigated in the following.

Figure 4 illustrates that the confinement improvement factor $H_{98}(y, 2)$ correlates with a deeper $E_{r}$ well. In the plot, data from Charge Exchange Recombination Spectroscopy (CXRS) and Doppler Reflectometry (DR) are combined. For convenience $\left|E_{r \text {,min }}\right|$ is shown. L-mode (black) and H-mode data (blue, favorable configurations) are well separated. L-mode 


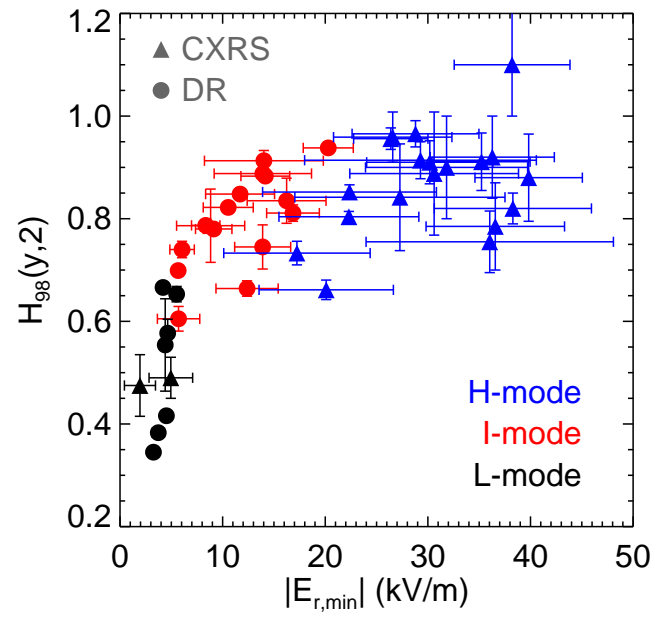

FIG. 4: Confinement improvement factor $H_{98}(y, 2)$ versus absolute radial electric field $E_{r}$ minimum for L-mode, I-mode and $\mathrm{H}$-mode. I-mode fills the region between L-mode and $\mathrm{H}$-mode in both $H_{98}$ and $\min \left(E_{r}\right)$. CXRS data taken from Ref. [22].

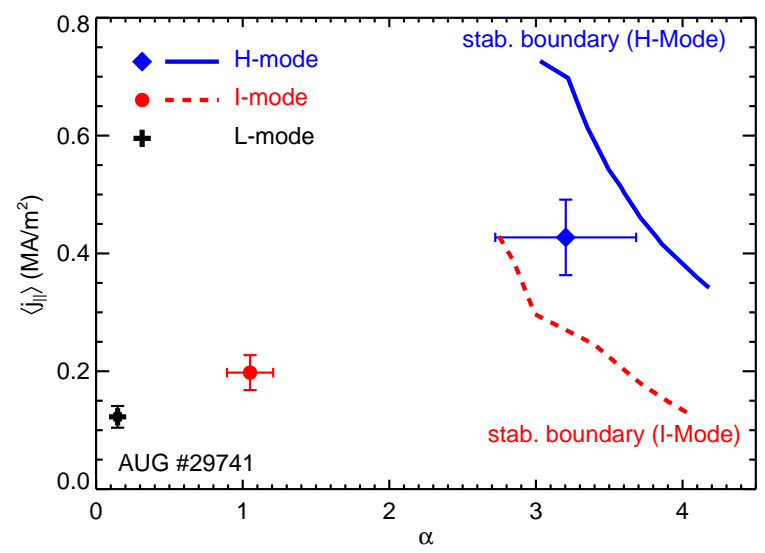

FIG. 5: Peeling-ballooning stability diagram showing results for Lmode (black), I-mode (red) and H-mode (blue). The experimental value is given by the points while the respective stability boundaries are given as lines. While in H-mode, the experimental point is close to the stability boundary, the I-mode is peeling-balloning stable. For L-mode, no stability boundary is found, even when increasing the pedestal parameters by a factor of three.

radial electric field minima can attain values up to $-5 \mathrm{kV} / \mathrm{m}$, while $\mathrm{H}$-modes lie in the range -15 to $-40 \mathrm{kV} / \mathrm{m}$. The gap between the two confinement regimes is filled with I-mode data points. While this gap is reasonably well defined for the $E_{r}$ values, it is not as clear for the confinement improvement factor $H_{98}(y, 2)$ : here, L-mode and $\mathrm{H}$-mode data points are also mostly separated, but the I-mode overlaps significantly with $\mathrm{H}$-mode in the range $H_{98}(y, 2)=0.7-0.9$.

It has been shown for Alcator C-Mod that the I-mode is peeling-ballooning stable, i.e. type-I ELMs do not occur $[5,6]$. Figure 5 shows an MHD stability diagram calculated with the MishKa code [31] for an AUG discharge comparable to the one above (AUG \#29741), also going from L- mode into I-mode and then into H-mode. The plot is described by the normalized pressure gradient $\alpha$, following the definition of Ref. [32], and the flux-surface averaged edge current density $\left\langle j_{\|}\right\rangle$. The lines show the stability boundaries for Iand H-modes. Following Ref. [33], they denote the boundary where $\gamma / v_{\mathrm{A}}=0.04$ is fulfilled. Here, $\gamma$ is the growth rate for different toroidal mode numbers $n$ and $v_{\mathrm{A}}$ is the Alfvén speed. This criterion robustly determines the region where mode growth is found. For L-mode, no stability boundary is found, even when increasing the pedestal parameters by a factor of three. Above and to the right of the boundaries peelingballooning modes are unstable, i.e. ELMs should occur if the edge parameters are in that region. Within uncertainties, the H-mode plasma edge parameters are close to the stability boundary. This agrees with the experimental observation of type-I ELMs in the H-mode phase of the discharge. In contrast, both the L-mode and I-mode parameters are deeply in the stable region of the diagram. Hence, just as the L-mode, the I-mode is peeling-ballooning stable, and no type-I ELMs are expected, which is consistent with the I-modes found so far in ASDEX Upgrade.

\section{DENSITY TURBULENCE BURSTS}

In ASDEX Upgrade, the I-mode edge exhibits strong and intermittent density fluctuation bursts, which are observed by several diagnostics [13, 34]. While the background density turbulence level is reduced in I-mode compared to L-mode, these bursts can have fluctuation amplitudes stronger than those in L-mode. They last for roughly 2-10 $\mu$ s and they have been shown to be connected to the WCM. In Ref. [34], structures of roughly $5 \mathrm{~mm}$ size have been investigated and a generation mechanism including the radial temperature gradient has been suggested. Extending these previous studies, the properties of these bursts at larger sizes and their observation by other diagnostics will be presented in the following.

\section{A. Characterization}

Figure 6 depicts for comparison two turbulence amplitude time traces of $10 \mathrm{~ms}$ length measured with Doppler reflectometry in the L-mode phase (a) and in the I-mode phase (b) of a plasma discharge. The Doppler reflectometer was used at fixed frequency, measuring at $\rho_{\text {pol }} \approx 0.99$, which is close to the $\mathrm{v}_{\perp}$ minimum, as observed in the comparable discharge of Figs. 2 and 4. The perpendicular wavenumber of the density fluctuations probed is $k_{\perp}=12 \mathrm{~cm}^{-1}$. In the L-mode (Fig. 6(a)), there is a background fluctuation level, and individual fluctuation events are comparably regular and of low amplitude. In contrast, in the I-mode (Fig. 6(b)), the fluctuation amplitude is in general lower, and it exhibits strong irregularly spaced bursts, which are significantly stronger than any fluctuations in L-mode (a). These intermittent events last about $2-10 \mu$ s.

Figure 7 shows a contour plot of the temporal evolution of the probability density function (PDF) from L-mode, through 


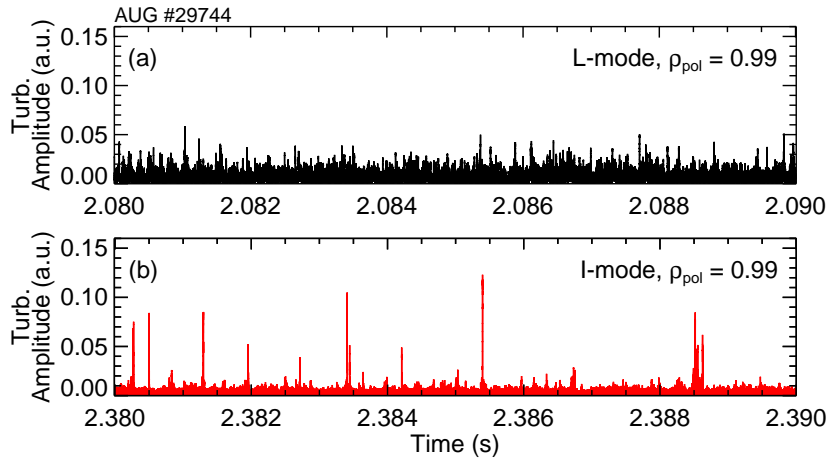

FIG. 6: Comparison of turbulence amplitude behavior in (a) L-mode and (b) I-mode. In I-mode, a low turbulence level and strong bursts are observed, while the L-mode exhibits a higher turbulence level. Reprinted with permission from [34].

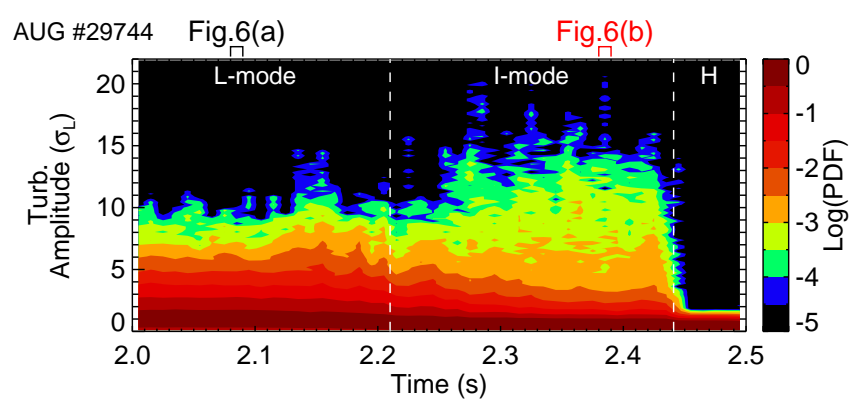

FIG. 7: Temporal evolution of the probability density function from L-mode through I-mode into H-mode. The time windows from Fig. 6(a) and (b) are indicated on top. Reprinted with permission from [34].

the I-mode, and into H-mode. The fluctuation amplitudes have been normalized to the value of the L-mode standard deviation $\sigma_{\mathrm{L}}$. Two dominant effects can be observed: The strong intermittent density bursts cause a tail of the PDF at values above $10 \sigma_{\mathrm{L}}$. In contrast, lower turbulence amplitudes are effectively reduced in I-mode. The fact that the occurrence of fluctuations at comparably small amplitudes is reduced while the occurrence of fluctuations at large amplitudes is increased is an interesting observation, and will be further investigated below.

As stated above, the WCM is an intrinsic feature of the Imode in Alcator C-Mod [11, 35] and AUG [12]. Fig. 8(a) shows three time traces of the Doppler reflectometer amplitude signal relative to the time when a burst arrives $(t=0)$ for different I-mode discharges. They differ in that the heating method is ECRH only (2.2 MW) in \#30865 and a combination of ECRH (0.7 MW) and NBI (2.0 MW) in \#29741 and \#29744. In each case, events can be observed just before the last density burst occurs. The temporal separation of the precursor events $\Delta t$ corresponds to a frequency of roughly $f=90 \mathrm{kHz}$. Figure 8(b) depicts the three corresponding spectra from conventional reflectometry, where the WCM can be seen. In all cases, $f_{\mathrm{WCM}} \approx 100 \mathrm{kHz}$, which is comparable to the precursor frequency in (a). The small difference is due to slightly different radial measurement locations of the two
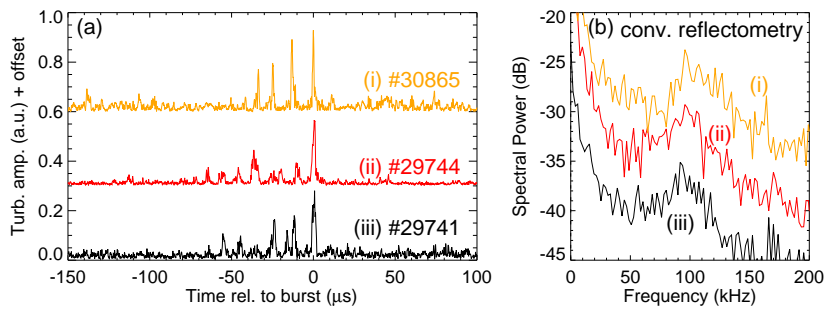

FIG. 8: (a) Turbulence amplitude signal from Doppler reflectometry showing precursor activity connected to the intermittent density bursts. The precursor frequency $1 / \Delta t$, where $\Delta t$ is the time between precursor events, corresponds to the WCM frequency observed by conventional reflectometry (b). ECRH only (\#30865) and ECRH+NBI (\#29741, \#29744) shown.

diagnostics. The above indicates a possible connection between WCM and the bursts reported here. However, a causal connection between WCM and bursts cannot be shown here, since no energy transfer measurements are available with the current AUG confinement region turbulence diagnostics.

Assuming a linear dispersion relation $\omega=v k$, the WCM frequency $f_{\mathrm{WCM}}$ in Fig. 8 (b) can be translated into a corresponding wavenumber by $k_{\mathrm{WCM}}=2 \pi f_{\mathrm{WCM}} / \mathrm{v}_{\perp}$, where $\mathrm{v}_{\perp}$ is the perpendicular velocity of density fluctuations. For all cases, this yields values of $k_{\mathrm{WCM}} \approx 1.0-1.5 \mathrm{~cm}^{-1}$, which is comparable to the value obtained from measurements at Alcator C-Mod $\left(k_{\mathrm{WCM}}=1.3 \pm 0.5 \mathrm{~cm}^{-1}\right)$ [11]. Hence, the wavenumber ranges of the WCM and the bursts are substantially different. One may speculate that a reason why the WCM is nonetheless reflected at high $k_{\perp}$ could be that the WCM modulates the turbulence level of the small-scale structures. Another possible explanation is that the WCM becomes asymmetric due to wave steepening, and thus higher $k_{\perp}$ are contained in the spectrum of the WCM. In order to assess the importance of different structure sizes, measurements at different $k_{\perp}$ are presented in the following.

\section{B. Dependence on structure size}

The density bursts presented above have been measured at a comparably high wavenumber, $k_{\perp} \approx 12 \mathrm{~cm}^{-1}$, i.e. small structure sizes of roughly $0.5 \mathrm{~cm}$ wavelength. Typically, density transport is assumed to be dominated by larger structures [36], which generally also have higher amplitudes. In order to investigate the existence of the intermittent I-mode density fluctuations at larger scales, specific discharges comparable to the one presented in Fig. 2 have been performed. The perpendicular wavenumber measured with the Doppler reflectometer has been scanned between $k_{\perp} \approx 5-10 \mathrm{~cm}^{-1}$, extending the previously investigated scales substantially. As in Fig. 2, Doppler reflectometer measurement locations are in the $E_{r}$ minimum $\left(\rho_{\text {pol }} \approx 0.995\right)$. Due to the density gradient, they have a radial extent of roughly $2 \mathrm{~mm}$ [37].

Figure 9 shows probability density functions (PDFs) of the turbulence amplitude at different $k_{\perp}$. In Fig. 9(a), the evolution of the PDF during increasing I-mode confinement is depicted 

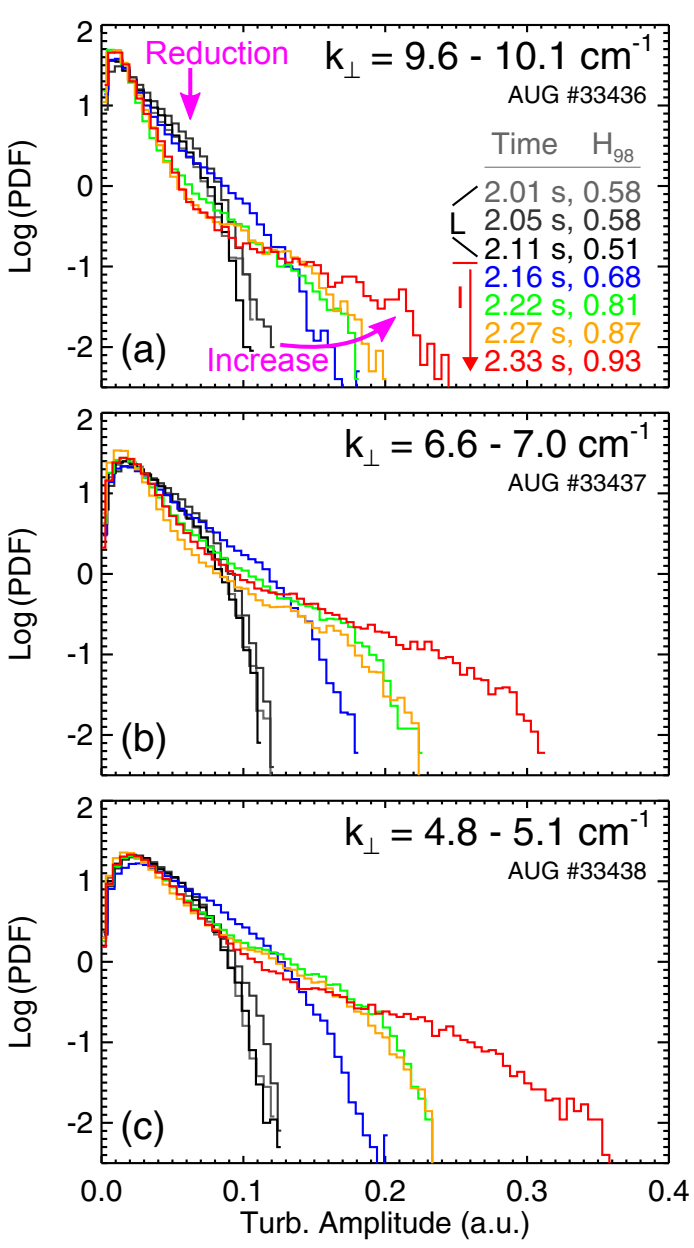

FIG. 9: PDFs of density fluctuation amplitudes obtained at $\rho_{\text {pol }}=$ 0.99 and for different structure sizes, $k_{\perp} \approx 10 \mathrm{~cm}^{-1}(\mathrm{a}), k_{\perp} \approx 7 \mathrm{~cm}^{-1}$ (b), $k_{\perp} \approx 5 \mathrm{~cm}^{-1}$ (c). For all structure scales, the PDF develops a heavy tail in I-mode at increasing $H_{98}(y, 2)$, which reflects the observation of increased density fluctuation events.

for a perpendicular wavenumber of $k_{\perp} \approx 10 \mathrm{~cm}^{-1}$. The legend indicates both the time point during the discharge and the corresponding confinement improvement factor $H_{98}(y, 2)$. Note that for 2.01-2.11 s, the plasma is still in L-mode, which is used as the reference. The trend seen in Fig. 6(c) is recovered: as the plasma enters the I-mode, the PDF becomes broader while it decreases at low fluctuation amplitudes (blue line). During improving I-mode confinement, there is a reduction of amplitudes between 0.03 and 0.08 (arbitrary units), while those at higher amplitudes $(>0.08)$ increase. The structures with the largest fluctuation amplitudes are observed during the best confinement of $H_{98}(y, 2)=0.93$, consistent with results presented above and in a previous study on AUG [34].

In Fig. 9(b), larger structures have been measured by using a different angle of incidence of the Doppler reflectometer. Although the discharge is subsequent to the one presented in (a), the legend is omitted since $H_{98}(y, 2)$ differs by 0.02 at most, which underlines the good reproducibility of the discharge. For the L-mode case, the PDF is broader at small amplitudes than in Fig. 9(a). This shows that more turbulence power is at smaller wavenumbers, which is a well-known fact from decades of density turbulence measurements [38]. Intermittent density events are also observed at this $k_{\perp}$ window with amplitudes even larger than in the higher $k_{\perp}$ window in Fig. 9(a). This trend is continued in Fig. 9(c) $\left(k_{\perp} \approx 5 \mathrm{~cm}^{-1}\right)$, where the PDF also develops a strong tail toward large amplitudes in I-mode. Note that also the PDF is broader at low amplitudes, indicating more turbulence power than at smaller scales (Figs. 9(a) and (b)).

Building on previous results [34], it can be stated that the strong and intermittent density events are not only seen when small structures are probed, but also at comparably large structures, $k_{\perp} \approx 5 \mathrm{~cm}^{-1}$, which corresponds to structures larger than $1 \mathrm{~cm}$. At large structure sizes they are even more pronounced.

\section{Temporal evolution}

In order to study the influence of the observed bursts on density transport, the above observations are compared to other diagnostics which preferably measure in the scrapeoff layer or the divertor. The time-delay between the occurence of the burst in the confinement region and its impact in the SOL/divertor can give information on where the bursts are generated. To study the causality of the observed bursts, related measurements from absolute extended ultraviolet (AXUV) diode based bolometer diagnostic [39] are analyzed. The AXUV bolometer channels have a temporal resolution of $5 \mu \mathrm{s}$ and are sensitive to radiation in the range of $1 \mathrm{eV}-10 \mathrm{keV}$. The measurement depends on a combination of density, temperature, and impurity concentration. There are 256 channels installed at AUG, covering the whole poloidal cross-section. These characteristics make the AXUV bolometers well suited to serve as a two-dimensional comparison with other measurements, in this case the intermittent density bursts observed by Doppler reflectometry.

Figure 10 shows the poloidal cross section of AUG including the plasma equilibrium. Solid grey lines are closed flux surfaces and show the contours of constant normalized poloidal flux between $\rho_{\text {pol }}=0.1$ and 1.0 (separatrix), spaced evenly in 10 levels. The black cross indicates the measurement of the Doppler reflectometer, which is at $\rho_{\text {pol }} \approx 0.99$. Furthermore, selected lines of sight (LOS) of the AXUV bolometer analyzed in Fig. 11 are indicated. It should be noted that the bolometer channels are toroidally separated from the Doppler reflectometer by approximately $130^{\circ}$. The setup allows time delays between characteristic events to be estimated. Moreover, channel DVC 3 (orange) will be used to determine whether the bolometry is sensitive to the bursts in the confinement region. Since turbulent structures are normally elongated along the magnetic field lies with $k_{\|} \ll k_{\perp}$, it is not expected that the toroidal separation should play a significant role in the following analysis. Apart from diagnostic geometry, the connection between a position close to the DR measurement but in the SOL at $\rho_{\text {pol }}=1.01$ and the divertor is indicated. The connection length is $L_{\mathrm{c} 1} \approx 12 \mathrm{~m}$ (blue, low field side (LFS)). 


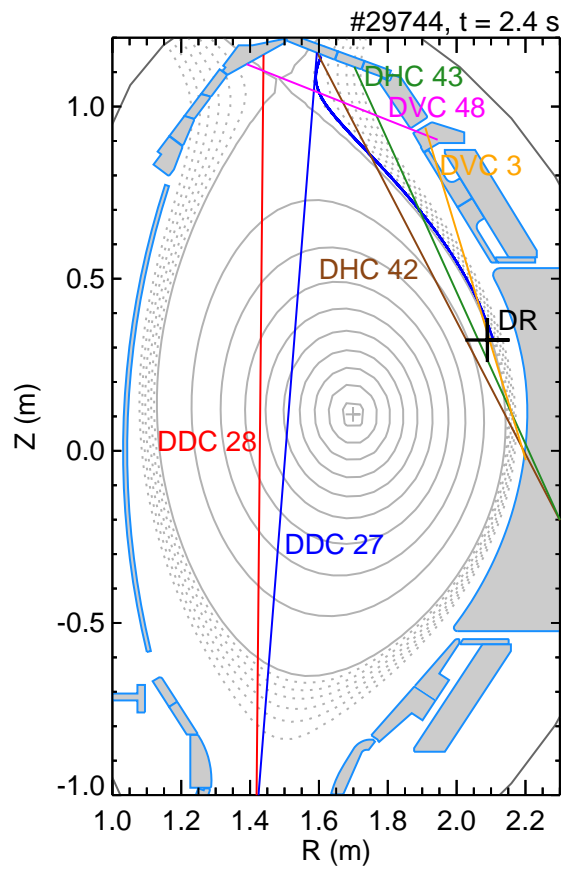

FIG. 10: Plasma geometry with Doppler reflectometer measurement location (DR) and AXUV bolometer lines of sight indicated. The respective signals are shown in Fig. 11.

Three subsequent turbulence bursts from discharge \#29744 starting at $t_{0}=2.43535 \mathrm{~s}$ are depicted in Fig. 11 as observed by Doppler reflectometry and the bolometer channels mentioned above. In (a), the three bursts measured by Doppler reflectometry exhibit fluctuation levels significantly above the background level. Just before the main bursts, the smaller precursor events which have the frequency of the WCM are seen as in Fig. 8. Vertical dotted and dashed lines indicate the onset of the precursor and the maximum of the turbulence burst, respectively. In Fig. 11(b), time traces of the bolometer lines DDC 27 (outer divertor leg) and DDC 28 (inner divertor leg) show a clear correlation to the turbulence amplitude signal. A time delay of roughly $45 \mu \mathrm{s}$ is detected with respect to the edge fluctuation amplitude, both of onset and of maximum value of the two divertor bolometer channels. The time delay for the maximum of the second burst in Fig. 11 is indicated examplarily by the horizontal arrows. The connection length is roughly $12 \mathrm{~m}$ for the outer divertor (indicated in blue in Fig. 10). Since the LOS of DDC 27 and 28 go through the whole plasma (cf. Fig. 10), the time traces of two additional channels (DHC 42 and 43), going through the plasma edge and monitoring the upper divertor, are shown in Fig. 11(c). Also in these channels, the radiation increase related to the density bursts is clearly visible. It is interesting to note that the channel observing the outer strike line (DHC 42, multiplied by a factor 3 in the figure) shows substantially less signal increase than the channel further outside (DHC 43). This could be due to local effects in the divertor, which increase the radiation and are triggered by the turbulence burst.

Figure 11(d) shows a LOS which measures exclusively the divertor (DVC 48), and one LOS which does not observe the


FIG. 11: Zoom to three subsequent density bursts starting at $t_{0}=2.43535 \mathrm{~s}$ as observed by Doppler reflectometry (a) and bolometry (b-d). The different bolometer lines of sight are indicated in the poloidal cross-section in Fig 10.

divertor at all, but crosses the position of the Doppler reflectometer measurement (DVC 3). While DVC 3 shows no clear correlation to the density bursts from (a), DVC 48 is strongly affected by them, again with a time delay of roughly $45 \mu \mathrm{s}$. This shows that even though the density bursts observed by Doppler reflectometry in the edge region have high amplitudes, they are still not detected by bolometry directly and in the confinement region. Instead it is rather the effect of the bursts in the divertor which gives a strong response in the bolometry signal. All bolometer LOS except DVC 3 show a net increase in the time window shown in Fig. 11. This is due to the fact that the time between the density bursts is comparably short (about $225 \mu \mathrm{s}$ ), and the radiation event in the divertor has not yet completely subsided when the next burst arrives. Hence, the radiation due to the bursts accumulates. About $700 \mu$ s after the time window shown in Fig. 11, the bolometer signal has returned to the level at the beginning of Fig. 11.

The fact that the bolometer measurement is a combination of density, temperature, and impurity concentration prohibits a clear statement on the origin of the bolometer signal increase. However, the squared density dependence of the bolometer signal indicates that the events are most likely caused by a density increase in the divertor.

The time delay $\Delta t$ between turbulence burst observation 
and divertor response has to be compared to the ion sound speed $c_{s} \approx \sqrt{T_{e} / m_{i}}$. In order to calculate a corresponding parallel velocity of the structures, it is assumed that the density burst leaves the confined plasma at the height of the Doppler reflectometer measurement position. The low-fieldside connection length calculated from $1 \mathrm{~cm}$ outside the separatrix to the divertor is $L_{c 1} \approx 12 \mathrm{~m}$. This gives a velocity of $\mathrm{v}=12 \mathrm{~m} / 45 \mu \mathrm{s} \approx 270 \mathrm{~km} / \mathrm{s}$, which is four times the ion sound speed at an assumed electron temperature of $T_{e}=90 \mathrm{eV}$. However, since the Doppler reflectometer measurement is local, the density burst could have occurred earlier at another toroidal and poloidal position, such that the present consideration only gives an upper limit to the parallel velocity. In this context, it should be noted that while all bursts observed by Doppler reflectometry are also observed by the bolometer, the opposite is not true. This is probably due to the toroidal and poloidal localization of the Doppler reflectometer measurement. Nevertheless, the above shows that the density burst is observed first inside the separatrix, and later in the divertor. Hence the burst has been generated in the plasma edge [34], and not in the divertor. This is important in particular considering that the bursts are connected to the WCM, both of which are related exclusively to the confined plasma of the I-mode regime.

\section{SUMMARY AND CONCLUSIONS}

An overview of recent observations related to the I-mode confinement regime on the ASDEX Upgrade tokamak has been given. It has been shown that there is a weak toroidal magnetic field strength dependence $\left(\propto B_{t}^{0.39}\right)$ of the L-I transition power threshold $P_{\mathrm{L}-\mathrm{I}}$. Based on this observation, it is unclear whether the physics behind the L-I transition and the L-H transition could have commonalities. Nevertheless, after the transition to I-mode, the edge radial electric field $\left(E_{r}\right)$ well increases in magnitude, which is correlated with a stronger $E_{r}$ shear, as is observed generally in H-mode. The increased $E_{r}$ shear is also correlated with the confinement improvement factor $H_{98}(y, 2)$. In contrast to $\mathrm{H}$-mode, the I-mode is peelingballooning stable, as shown through stability analysis using the Mishra code.

There is a pronounced reduction of low fluctuation amplitude activity. However, during the I-mode, strong density turbulence bursts are observed, which are stronger than L-mode fluctuations. These I-mode density bursts cause a heavy-tail PDF. Furthermore, they are connected to the weakly coherent mode (WCM), as seen in the time delay of precursor events. They exist at all structure sizes investigated $\left(k_{\perp}=4.8-\right.$ $\left.12.0 \mathrm{~cm}^{-1}\right)$ and the largest structures $\left(k_{\perp} \approx 5 \mathrm{~cm}^{-1}\right)$ show the strongest amplitudes. Furthermore, the intermittent events are observed several tens of microseconds later in the divertor. The combination of strong density perturbation amplitudes, even at large sizes, and the divertor impact, is an indication that the bursts could indeed play a role in inhibiting the edge density pedestal to develop. The link to the WCM underlines the intimate connection of the density bursts to the I-mode confinement regime.

\section{Acknowledgments}

This work was partly performed in the framework of the Helmholtz Virtual Institute on plasma dynamical processes and turbulence using advanced microwave diagnostics ( $\mathrm{VH}-$ VI-526) and within the framework of the EUROfusion Consortium and has received funding from the Euratom research and training programme 2014-2018 under grant agreement No 633053. Furthermore, funding from the EUROfusion Enabling Research work-package AWP15-ENR-09/IPP-02 is acknowledged. The views and opinions expressed herein do not necessarily reflect those of the European Commission.
[1] F. Wagner, G. Becker, K. Behringer, D. Campbell, A. Eberhagen, W. Engelhardt, G. Fussmann, O. Gehre, J. Gernhardt, G. v. Gierke, G. Haas, M. Huang, F. Karger, M. Keilhacker, O. Klber, M. Kornherr, K. Lackner, G. Lisitano, G. G. Lister, H. M. Mayer, D. Meisel, E. R. Mller, H. Murmann, H. Niedermeyer, W. Poschenrieder, H. Rapp, and H. Röhr, Phys. Rev. Lett. 49, 1408 (1982).

[2] F. Wagner, Plasma Phys. Control. Fusion 49, B1 (2007).

[3] D. G. Whyte, A. E. Hubbard, J. W. Hughes, B. Lipschultz, J. E. Rice, E. S. Marmar, M. Greenwald, I. Cziegler, A. Dominguez, T. Golfinopoulos, N. Howard, L. Lin, R. M. McDermott, M. Porkolab, M. L. Reinke, J. Terry, N. Tsujii, S. Wolfe, S. Wukitch, Y. Lin, and the Alcator C-Mod Team, Nucl. Fusion 50, 105005 (2010).

[4] F. Ryter and et al., Nucl. Fusion (2016), accepted for publication.

[5] J. Hughes, P. Snyder, J. Walk, E. Davis, A. Diallo, B. LaBombard, S. Baek, R. Churchill, M. Greenwald, R. Groebner, A. Hubbard, B. Lipschultz, E. Marmar, T. Osborne, M. Reinke, J. Rice, C. Theiler, J. Terry, A. White, D. Whyte, S. Wolfe, and X.
Xu, Nucl. Fusion 53, 043016 (2013).

[6] J. R. Walk, J. W. Hughes, A. E. Hubbard, J. L. Terry, D. G. Whyte, A. E. White, S. G. Baek, M. L. Reinke, C. Theiler, R. M. Churchill, J. E. Rice, P. B. Snyder, T. Osborne, A. Dominguez, and I. Cziegler, Phys. Plasmas 21, 056103 (2014).

[7] B. Sieglin, T. Eich, A. Scarabosio, G. Arnoux, I. Balboa, S. Devaux, A. Herrmann, F. Hoppe, M. Hlzl, A. Kallenbach, P. Lang, G. F. Matthews, S. Marsen, S. Pamela, M. Rack, R. Wenninger, the ASDEX Upgrade Team, and J. E. Contributors, Plasma Phys. Control. Fusion 55, 124039 (2013).

[8] E. Righi, D. Bartlett, J. Christiansen, G. Conway, J. Cordey, L.G. Eriksson, H. D. Esch, G. Fishpool, C. Gowers, J. de Haas, P. Harbour, N. Hawkes, J. Jacquinot, T. Jones, W. Kerner, Q. King, C. Lowry, R. Monk, P. Nielsen, F. Rimini, G. Saibene, R. Sartori, B. Schunke, A. Sips, R. Smith, M. Stamp, D. Start, K. Thomsen, B. Tubbing, and N. Zornig, Nuclear Fusion 39, 309 (1999).

[9] F. Ryter, S. Rathgeber, L. B. Orte, M. Bernert, G. Conway, R. Fischer, T. Happel, B. Kurzan, R. McDermott, A. Scarabosio, W. Suttrop, E. Viezzer, M. Willensdorfer, E. Wolfrum, and the 
ASDEX Upgrade Team, Nucl. Fusion 53, 113003 (2013).

[10] A. E. Hubbard, T. Osborne, F. Ryter, M. Austin, L. Barrera Orte, R. M. Churchill, I. Cziegler, M. Fenstermacher, R. Fischer, S. Gerhardt, R. Groebner, P. Gohil, T. Happel, J. W. Hughes, A. Loarte, R. Maingi, P. Manz, A. Marinoni, E. S. Marmar, R. McDermott, G. McKee, T. Rhodes, J. Rice, L. Schmitz, C. Theiler, E. Viezzer, J. R. Walk, A. White, D. Whyte, S. Wolfe, E. Wolfrum, Z. Yan, and the Alcator C-Mod, ASDEX Upgrade and DIII-D Teams, Nucl. Fusion (2016), accepted.

[11] I. Cziegler, P. H. Diamond, N. Fedorczak, P. Manz, G. R. Tynan, M. Xu, R. M. Churchill, A. E. Hubbard, B. Lipschultz, J. M. Sierchio, J. L. Terry, and C. Theiler, Phys. Plasmas 20, 055904 (2013).

[12] P. Manz, P. Lauber, V. Nikolaeva, T. Happel, F. Ryter, G. Birkenmeier, A. Bogomolov, G. Conway, M. Manso, M. Maraschek, D. Prisiazhniuk, and E. Viezzer, Nucl. Fusion 55, 083004 (2015).

[13] F. Ryter, W. Suttrop, B. Brüsehaber, M. Kaufmann, V. Mertens, H. Murmann, A. G. Peeters, J. Stober, J. Schweinzer, H. Zohm, and the ASDEX Upgrade Team, Plasma Phys. Control. Fusion 40, 725 (1998).

[14] A. Marinoni, J. Rost, M. Porkolab, A. Hubbard, T. Osborne, A. White, D. Whyte, T. Rhodes, E. Davis, D. Ernst, and K. Burrell, Nucl. Fusion 55, 093019 (2015).

[15] A. Hubbard, D. Whyte, R. Churchill, A. Dominguez, J. Hughes, Y. Ma, E. Marmar, Y. Lin, M. Reinke, and A. White, Nucl. Fusion 52, 114009 (2012).

[16] L. Cupido, S. Graca, G. D. Conway, M. Manso, F. Serra, and A. U. Team, Rev. Sci. Instrum. 77, 10E915 (2006).

[17] Y. R. Martin, T. Takizuka, and the ITPA CDBM H-mode Threshold Database Working Group, J. Phys.: Conf. Ser. 123, 012033 (2008).

[18] M. Hirsch, E. Holzhauer, J. Baldzuhn, B. Kurzan, and B. Scott, Plasma Phys. Control. Fusion 43, 1641 (2001).

[19] G. R. McKee, R. J. Fonck, D. K. Gupta, D. J. Schlossberg, M. W. Shafer, R. L. Boivin, and W. Solomon, Plasma Fus. Res. 2, S1025 (2007).

[20] T. Estrada, T. Happel, L. Eliseev, D. López-Bruna, E. Ascasíbar, E. Blanco, L. Cupido, J. M. Fontdecaba, C. Hidalgo, R. Jiménez-Gómez, L. Krupnik, M. Liniers, M. E. Manso, K. J. McCarthy, F. Medina, A. Melnikov, B. v. Milligen, M. A. Ochando, I. Pastor, M. A. Pedrosa, F. L. Tabarés, D. Tafalla, and the TJ-II Team, Plasma Phys. Control. Fusion 51, 124015 (2009).

[21] H. W. Müller, J. Adamek, R. Cavazzana, G. D. Conway, J. P. Gunn, A. Herrmann, J. Horacek, C. Ionita, M. Kocan, M. Maraschek, C. Maszl, F. Mehlmann, B. Nold, M. Peterka, V. Rohde, R. Schrittwieser, N. Vianello, E. Wolfrum, M. Zuin, and the ASDEX Upgrade Team, Fluctuations, ELM Filaments and Turbulent Transport in the SOL at the Outer Midplane of AS-
DEX Upgrade (IAEA, Daejon, Korea, 2010), report EXD/P323.

[22] E. Viezzer, T. Pütterich, G. D. Conway, R. Dux, T. Happel, J. C. Fuchs, R. M. McDermott, F. Ryter, B. Sieglin, W. Suttrop, M. Willensdorfer, E. Wolfrum, and the ASDEX Upgrade Team, Nucl. Fusion 53, 053005 (2013).

[23] G. D. Conway, C. Angioni, R. Dux, F. Ryter, A. G. Peeters, J. Schirmer, C. Troester, CFN Reflectometry Group, and the ASDEX Upgrade Team, Nucl. Fusion 46, S799 (2006).

[24] L. Vermare, P. Hennequin, O. D. Gurcan, C. Bourdelle, F. Clairet, X. Garbet, R. Sabot, and the Tore Supra Team, Phys. Plasmas 18, 012306 (2011).

[25] T. Happel, A. Bañón Navarro, G. D. Conway, C. Angioni, M. Bernert, M. Dunne, E. Fable, B. Geiger, T. Görler, F. Jenko, R. M. McDermott, F. Ryter, U. Stroth, and the ASDEX Upgrade Team, Phys. Plasmas 22, 032503 (2015).

[26] K. Ida, Plasma Phys. Control. Fusion 40, 1429 (1998).

[27] P. Sauter, T. Pütterich, F. Ryter, E. Viezzer, E. Wolfrum, G. Conway, R. Fischer, B. Kurzan, R. McDermott, S. Rathgeber, and the ASDEX Upgrade Team, Nucl. Fusion 52, 012001 (2012).

[28] H. Biglari, P. H. Diamond, and P. W. Terry, Phys. Fluids 2, 1 (1990).

[29] K. H. Burrell, Phys. Plasmas 4, 1499 (1997).

[30] P. W. Terry, Rev. Mod. Phys. 72, 109 (2000).

[31] A. Mikhailovskii, G. Huysmans, W. Kerner, and S. Sharapov, Plasma Phys. Rep. 23, 844 (1997).

[32] J. W. Connor, R. J. Hastie, H. R. Wilson, and R. L. Miller, Phys. Plasmas 5, 2687 (1998).

[33] A. Burckhart, M. Dunne, E. Wolfrum, R. Fischer, R. McDermott, E. Viezzer, M. Willensdorfer, and the ASDEX Upgrade Team, Nuclear Fusion 56, 056011 (2016).

[34] T. Happel, P. Manz, F. Ryter, P. Hennequin, A. Hetzenecker, G. Conway, L. Guimarais, C. Honoré, U. Stroth, E. Viezzer, and the ASDEX Upgrade Team, Nucl. Fusion 56, 064004 (2016).

[35] A. E. Hubbard, D. G. Whyte, R. M. Churchill, I. Cziegler, A. Dominguez, T. Golfinopoulos, J. W. Hughes, J. E. Rice, I. Bespamyatnov, M. J. Greenwald, N. Howard, B. Lipschultz, E. S. Marmar, M. L. Reinke, W. L. Rowan, J. L. Terry, and the Alcator C-Mod Group, Phys. Plasmas 18, 056115 (2011).

[36] M. Ramisch, N. Mahdizadeh, U. Stroth, F. Greiner, C. Lechte, and K. Rahbarnia, Phys. Plasmas 12, 032504 (2005).

[37] H.-J. Hartfuß and T. Geist, Fusion Plasma Diagnostics with mm-Waves (Wiley-VCH, Boschstr. 12, 69469 Weinheim, Germany, 2013).

[38] P. C. Liewer, Nucl. Fusion 25, 543 (1985).

[39] M. Bernert, T. Eich, A. Burckhart, J. C. Fuchs, L. Giannone, A. Kallenbach, R. M. McDermott, B. Sieglin, and the ASDEX Upgrade Team, Rev. Sci. Instrum. 85, 033503 (2014). 